\title{
Isometric Coding of Spiking Haptic Signals by Peripheral Somatosensory Neurons
}

\author{
Romain Brasselet $^{1}$, Roland S. Johansson ${ }^{2}$, and Angelo Arleo ${ }^{1, \star}$ \\ ${ }^{1}$ CNRS - UPMC Univ Paris 6, UMR 7102, F75005, Paris, France \\ Tel.: +33144272780 \\ angelo.arleo@upmc.fr \\ 2 UMEA Univ, Dept Integr Medical Biology SE-901 87 Umeå, Sweden
}

\begin{abstract}
We study how primary tactile afferents encode relevant contact features to mediate early processing of haptic information. In this paper, we apply metrical information theory to perform temporal decoding of human microneurography data. First, we enrich the theory by deriving a novel spike train metrics inspired by neuronal computation. This spike train metrics can be interpreted biologically and its behaviour is not influenced by spontaneous activity, which decreases the ability of other spike metrics to separate input patterns. Second, we employ our metrical information tools to demonstrate that primary spiking signals allow a putative neural decoder to go beyond stimulus discrimination. They transmit information about geometrical properties of the input space. We show that first-spike latencies are enough to guarantee maximum information transmission of tactile stimuli. However, entire primary spike trains are necessary to encode isometric representations of the stimulus space, a likely basis for generalisation in haptic perception.
\end{abstract}

Keywords: Temporal coding, spike-train metrics, information theory, isometric mapping, microneurography recordings, mechanoreceptors.

\section{Introduction}

We study neurotransmission at the early stages of the somatosensory pathway and focus on encoding/decoding of primary afferent tactile signals. We previously extended Shannon information theory to account for the metrical properties of spike time patterns when assessing neurotransmission reliability [2]3. Here we stress the importance for metrical information analysis to reflect the properties of an actual neural decoder - as opposed to an ideal observer - when studying the statistical dependence between stimulus and neural response. To do so, we derive a novel spike train metrics based on a parametric non-linear distance inspired by the probabilistic behaviour of the Spike-Response Model 5 . We apply the resultant metrical information theory to a data set of human microneurography recordings [6]. We perform a temporal decoding analysis of the responses of fingertip mechanoreceptors to a set of tactile stimulations. The main rationale beneath this work is to go beyond input discriminability and to capture possible

\footnotetext{
^ Corresponding author.

J. Cabestany, I. Rojas, and G. Joya (Eds.): IWANN 2011, Part I, LNCS 6691, pp. 528-536 2011.

(C) Springer-Verlag Berlin Heidelberg 2011
} 
geometrical regularities of the input space encoded by primary afferent spiking signals. We suggest that the ability to extract information about the geometrical organisation of the input depends on the characteristics of the neural decoder. We show that a properly tuned decoder can detect isometric mapping suitable for generalisation in haptic perception.

\section{Methods}

\subsection{Metrical Information Theory}

We define the metrical entropy $H^{*}(R)$ on the response space $R$ as:

$$
H^{*}(R)=-\sum_{r \in R} p(r) \log _{2}\left(\sum_{r^{\prime} \in R} p\left(r^{\prime}\right) \phi\left(r, r^{\prime}\right)\right)
$$

where the similarity measure $\phi\left(r, r^{\prime}\right)$ can be any real function with values in $[0,1]$. The conditional entropy given a set of stimuli $S$ can then be taken as:

$$
H^{*}(R \mid S)=\sum_{s \in S} p(s) H(R \mid s)=-\sum_{s \in S} \sum_{r \in R} p(r, s) \log _{2}\left(\sum_{r^{\prime} \in R} p\left(r^{\prime} \mid s\right) \phi\left(r, r^{\prime}\right)\right)
$$

Finally, similar to Shannon information theory [114], the metrical mutual information $I^{*}(R ; S)$ is the difference between marginal and conditional entropies:

$$
I^{*}(R ; S)=H^{*}(R)-H^{*}(R \mid S)=\sum_{s \in S} \sum_{r \in R} p(r, s) \log _{2}\left(\frac{\sum_{r^{\prime} \in R} p\left(r^{\prime} \mid s\right) \phi\left(r, r^{\prime}\right)}{\sum_{r^{\prime} \in R} p\left(r^{\prime}\right) \phi\left(r, r^{\prime}\right)}\right)
$$

If the event space consists of spiking signals we can take the similarity measure $\phi\left(r, r^{\prime}\right)$ as a decreasing function of the distance $D\left(r, r^{\prime}\right)$ between two spike trains $r, r^{\prime} \in R$. Henceforth, we define $\phi\left(r, r^{\prime}\right)$ as a Heaviside function of the distance:

$$
\phi\left(r, r^{\prime}\right)=\mathcal{H}\left(D_{c}-D\left(r, r^{\prime}\right)\right)
$$

where the critical distance $D_{c}$ is the cutoff parameter: for $D\left(r, r^{\prime}\right)<D_{c}$, responses $r, r^{\prime}$ are considered as identical, otherwise they are classified as different. If $D_{c}=0$ we recover the Shannon entropy from Eq. 1.

Optimality condition for neurotransmission. Akin to the principle of redundancy reduction in biological sensory processing [1], the metrical conditional entropy $H^{*}(R \mid S)$ constitutes a fundamental quantity for neurotransmission [9]. Optimal information transfer must both maximise $I^{*}(R ; S)$ and (at the same time) minimise $H^{*}(R \mid S)\left[3\right.$. In order to set the optimal parameter $D_{c}$ for $\phi\left(r, r^{\prime}\right)$ we consider two sets of $D\left(r, r^{\prime}\right)$ distances: (i) the distances between the responses elicited by the same stimulus - referred to as intra-stimulus distances; (ii) the distances between the responses elicited by different stimuli — named inter-stimulus distances. 
As the input spike waves across multiple afferents flow in the readout system, the relationship between intra- and inter-stimulus distance distributions evolves over time. Optimal discrimination occurs when the distributions of intra- and inter-stimulus distances stop overlapping, which implies that $H^{*}(R \mid S)$ is nil and $I^{*}(R ; S)$ is maximum [3]. The cut-off parameter $D_{c}$ corresponding to optimal neurotransmission can then be set as the distance at which the maximum intrastimulus distance becomes smaller than the minimum inter-stimulus distance. The time at which the critical distance $D_{c}$ can be determined indicates when perfect input discrimination occurs 3 .

Definition of a novel spike train metrics. Several parametric approaches have quantified the distance between spike trains (e.g. [13|12/10|8]). We previously employed the Victor-Purpura $\left(D_{V P}\right)$ distance 13 in order to define the similarity function $\phi\left(r, r^{\prime}\right)$, i.e. $D\left(r, r^{\prime}\right)=D_{V P}\left(r, r^{\prime}\right)$ in Eq. [4, and to embed spike metrics in our information theoretical analysis [2]3. In this paper we presents some results based on the Victor-Purpura distance (Sec. 3.2). In addition, we derive a novel spike train metrics to take into account the properties of a putative neural decoder (e.g. a population of downstream neurons along a processing pathway). We do that by defining a parametric non-linear spike train distance mimicking the Spike-Response Model (SRM) 5. When a SRM neuron receives an afferent spike train $s_{i}$, its membrane potential $V(t)$ is computed as:

$$
V(t)=\frac{1}{\tau} \sum_{i} \mathcal{H}\left(t-\hat{t}_{i}\right) \sqrt{t} e^{-\left(t-\hat{t}_{i}\right) / \tau}
$$

where $\mathcal{H}$ is the Heaviside function, $\hat{t}_{i}$ is the time of an input spike emitted by the presynaptic neuron $i$, and $\tau$ is a free parameter determining the decay time constant of the EPSP (excitatory post-synaptic potential) of the neuron. The discharge probability $p(t)$ is a sigmoid function of the membrane potential:

$$
p(t)=\frac{1}{1+e^{\left(V_{0}-V(t)\right) / \Delta V}}
$$

with $V_{0}$ denoting the probabilistic threshold potential. We can think of this probability of firing as a fundamental output of the SRM, because it does not depend on a particular trial but represents the way the signal is processed (on average). We thus define the distance between two spike trains $r_{1}, r_{2}$ in response to two stimuli $s_{1}, s_{2}$ as the difference between the corresponding firing probabilities:

$$
D_{S R M}\left(r_{1}, r_{2}\right)=\left(\int_{t}\left(p_{1}(t)-p_{2}(t)\right)^{z} d t\right)^{1 / z}
$$

with $z=2$ in this study. The definition of $D_{S R M}$ is reminiscent of that of Van Rossum distance [12] due to the filtering of the spike train with an exponential kernel function. However, we take into account a non-linearity, which is highly relevant because it captures the probabilistic threshold behaviour of a neuron in the present case, this is modelled with escape noise. An advantage of the SRM 
distance is that all the spikes do not contribute equally to it. Those that yield only subthreshold potentials have no significant contribution to the probability of spiking and thus do not contribute to the distance. Spikes evoking superthreshold potentials, induce large probabilities that may, in turn, contribute largely to the distance. Thus, the SRM distance can be employed to implement synchrony detection: two different spike trains whose interspike intervals are large may have a nil distance if the threshold is high. However, periods of intense discharge make the potential overcome the threshold and thus potentially increase the distance. This may be relevant in the case of spontaneous input activity that should not be taken into account. Distances such as Victor-Pupura and Van Rossum do not account for this property, i.e. they are always non-zero for spontaneous activities.

\section{Results}

\subsection{Metrical Analysis of Poisson Spike Trains}

We first compared the Victor-Purpura (VP), Van Rossum (VR) and SRM metrics in terms of their efficiency in assessing spike train separability. We simulated stochastic input spike trains generated according to a Poisson process with refractory period. At each time $t$, the effective probability of spiking was:

$$
p_{\text {eff }}(t)=\frac{p(t)}{1+e^{-t+\hat{t}+\tau_{\text {ref }}}}
$$

with $\hat{t}$ denoting the latest spike time, and $\tau_{\text {ref }}=5 \mathrm{~ms}$ the refractory period.

In a first protocol, we set the baseline firing probability to $p_{0}=0.01$. We simulated two stimuli: the first increased its probability of spiking from $p_{0}$ to 0.2 within the time window $300-600 \mathrm{~ms}$, whereas the second went from $p_{0}$ to 0.4 within $300-600 \mathrm{~ms}$. We then computed $n=400 \mathrm{VP}$, VR and SRM distances between pairs of spike trains from identical stimuli (i.e. intra-stimulus distances), as well as $n=400$ between pairs of spike trains from different stimuli (i.e. inter-stimulus distances). Figure 1A compares the distributions of inter- and intra-stimulus distances assessed according to VP, VR, and SRM measures after $600 \mathrm{~ms}$. The plots suggest, qualitatively, that the SRM distance captured better than VP and VR the separation between the two stimuli. To quantitatively assess the efficiency of stimulus separation, we computed the integral of the absolute difference between the histograms of intra- and inter-stimulus distances (i.e. if two histograms were identical, the efficiency would be nil; if they were non-overlapping, the efficiency would be maximum). The SRM efficiency was significantly larger than the two others (Fig. 1C), suggesting a better discriminability due to the smaller overlap between intra- and inter-stimulus distances.

In a second protocol, we set again the baseline probability to $p_{0}=0.01$ and we simulated two stimuli: the first increased its probability of spiking to 0.2 within the interval $300-500 \mathrm{~ms}$, whereas the second increased it to 0.2 within $350-550$ ms. Then, we computed $n=400 \mathrm{VP}, \mathrm{VR}$ and SRM distances between pairs of spike trains from identical stimuli, and $n=400$ between pairs of spike trains from different stimuli. Figures 1B, C show that again the SRM distance provided 

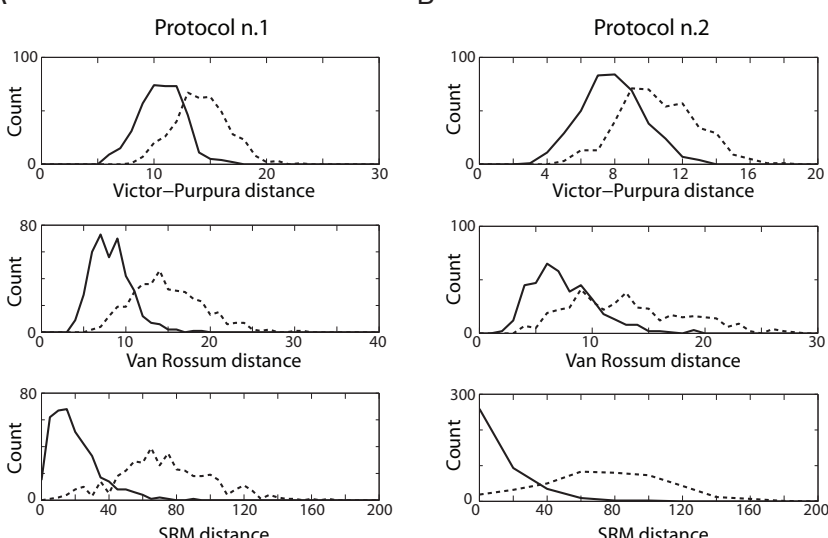

Van Rossum distance

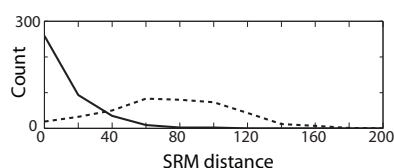

SRM distance
C

Stimulus separation efficiency

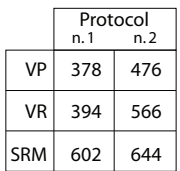

Fig. 1. Results. Comparative analysis between Victor-Purpura (VP), Van Rossum (VR), and SRM distances between two Poisson spike processes.

a better separation between the two stimuli compared to VP and VR distances. Note that, in this case, the efficiency of the SRM distance was not much larger than that of the VR distance (Fig. 1C). However, the SRM intra-stimulus distances were low compared to the inter-stimuli distances (Fig. 1B), which can be interpreted as a high signal-to-noise ratio (SNR) - since the level of intraand inter-stimulus distances can be understood as noise and signal, respectively. Thus, although in this example the efficiency of spiking pattern separation was only slightly improved by the SRM distance, the SNR significantly increased compared to VP and VR measures. If we defined the SNR as the mean interstimuli distance over the mean intra-stimulus distance, then the SNR would be $\approx 2$ for the $\mathrm{VR}$ distance and $\approx 4.5$ for the SRM distance.

\subsection{Isometric Coding of Human Microneurography Haptic Signals}

We applied our metrical information theory (Sec. 2.1) to a data set of human microneurography recordings of fingertip mechanoreceptor responses [6]. Mechanoreceptors innervate the epidermis and discharge according to mechanical indentations of the skin. The spike latencies of mechanoreceptor responses convey information about contact parameters faster than the fastest rate code, and fast enough to account for the use of tactile signals in natural manipulation 677. We focused on temporal coding of fast adapting (FA-I) mechanoreceptor activity [6]. The data set consisted of the responses of 42 FA-I units to 81 distinct tactile stimuli obtained by varying four contact parameters (Fig. 2A). The discharge of FA-I units (Figs. 2B,C) occurs rapidly with respect to stimulus onset and it is phasic (as opposed to other mechanoreceptor types which exhibit slow and tonic responses to skin indentation, [7]).

We first focused on the first spike latencies of FA-I responses. Each tactile stimulus corresponded to a single volley of spikes forming a spatiotemporal pattern 


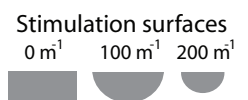

Stimulus orientation

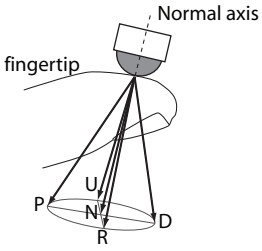

Force magnitude \& angle

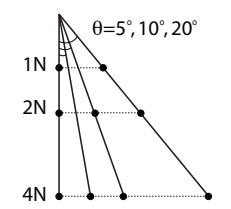

Force time course

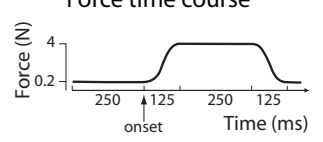

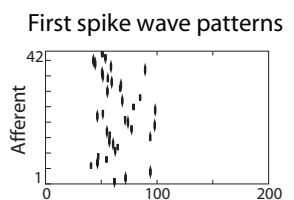
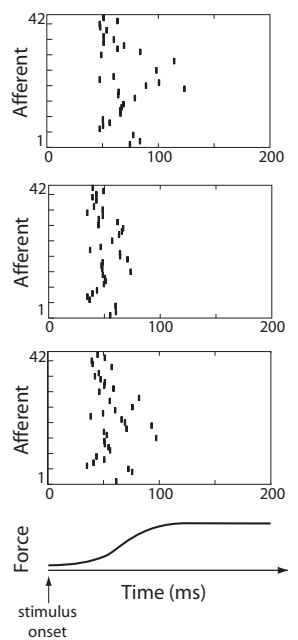

C
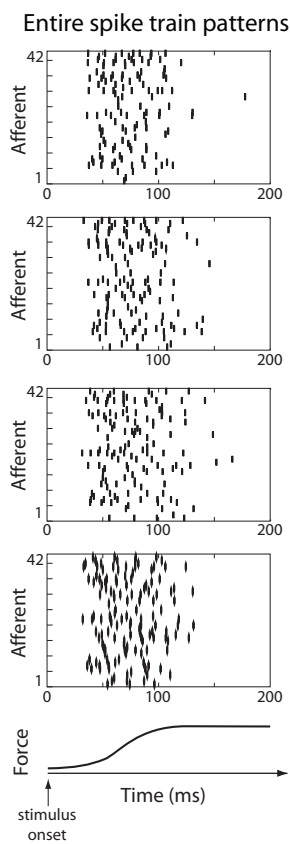

Fig. 2. Human microneurography recordings. (A) We considered 42 FA-I responses to 81 stimuli obtained by varying four contact parameters: probe curvature, force magnitude, force direction, and force angle relative to the normal direction. Black circles indicate available data for given force-angle combinations. (B,C) Samples of FA-I spikegrams when considering first spike waves only or entire spike train patterns, respectively.

(Fig. 2B). We subsequently accounted for the entire spike train patterns emitted by the population of 42 mechanoreceptors (Fig. 2C), to investigate the possible contribution of second and following spike waves to information transmission. We studied temporal encoding/decoding of mechanoreceptor discharges in terms of rapid and reliable discriminability -i.e. in terms of optimal information transfer as defined in Sec. 2.1. The results of Figure 3A compare the time course of metrical information $I^{*}(R ; S)$ (solid lines) and conditional entropy $H^{*}(R \mid S)$ (dashed lines) when considering first-spike waves (black lines) or full spike trains (grey curves). Perfect discrimination of 81 tactile stimuli based on the relative spike timing of mechanoreceptor responses occurred within $40 \mathrm{~ms}$ of the first spike emitted, i.e. very early in the protraction phase of stimulation (Fig. 3A, bottom). These results also suggested that taking into account entire spike trains did not provide any significant benefit in terms of discrimination capacity and velocity, corroborating the hypothesis on the relevance of the variability in the first-spike latency domain. To study the potential role of subsequent spikes we employed the Victor-Purpura metrics $D_{V P}$ to measure the distances between FA-I responses to a specific stimulus set. We computed the $D_{V P}$ between the responses to stimuli with force amplitudes 1 and $2 \mathrm{~N}$, i.e. $D_{V P}(1 N, 2 N)$, then to 


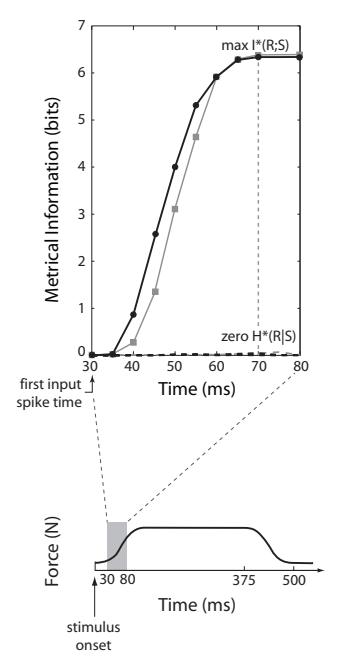

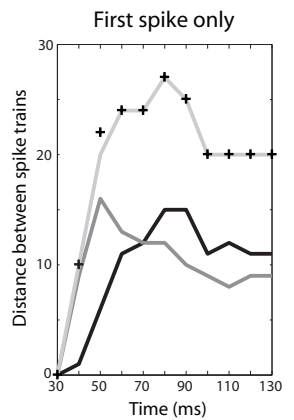

C

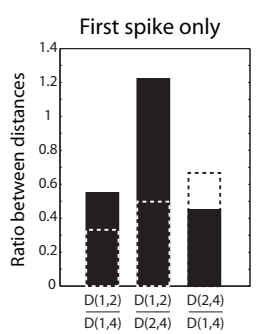

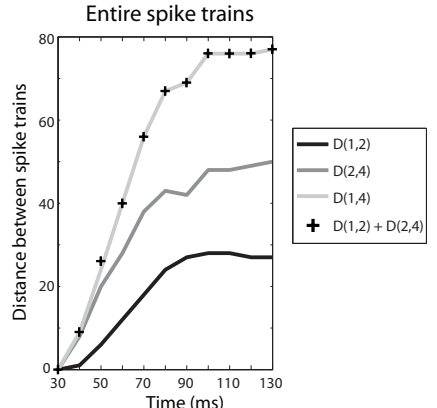

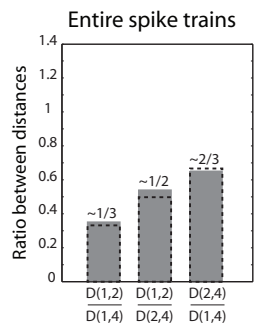

Fig. 3. Results. Metrical information analysis of 42 FA-I responses to 81 tactile stimuli. Each stimulus was presented 100 times. (A) Metrical information over time when considering either first spike waves only (black curve) or entire spike trains (grey line). (B) Time course of the distances between FA-I responses to stimuli with force amplitudes 1 and $2 \mathrm{~N}, D(1,2)$, then to stimuli with force 2 and $4 \mathrm{~N}, D(2,4)$, and finally to stimuli with force 1 and $4 \mathrm{~N}, D(1,4)$. For each combination, all the other contact parameters were varied. (C) Only entire spike trains allowed an isometric input-output mapping to be captured (dashed rectangles indicate the perfect isometric mapping).

stimuli with force 2 and $4 \mathrm{~N}$, i.e. $D_{V P}(2 N, 4 N)$, and finally to stimuli with force 1 and $4 \mathrm{~N}$, i.e. $D_{V P}(1 N, 4 N)$. Figure $3 \mathrm{~B}$ shows that the following relation was verified: $D_{V P}(1 N, 2 N)+D_{V P}(2 N, 4 N)=D_{V P}(1 N, 4 N)$. This result held when both only first spike waves (Fig. $3 \mathrm{~B}$, left) and entire spike trains were considered (Fig. 3B B right). Thus, in both cases the one-dimensional stimulus space was mapped onto a non-curved one-dimensional response space. This "alignment" property could be suitable to dissociate the problem of decoding the force of the stimulus from that of determining other features of the stimulus (i.e. pruning of the search state space). Importantly, Figure $3 \mathrm{C}$ shows that only when considering entire spike trains the distances between the responses tended to reflect the distances between the stimuli, suggesting an isometry-like property of the decoding measure. We further investigated this isometric coding property by analysing entire spike train responses to the stimulus set of Figure 4A, top. Decoding based on Victor-Purpura metrics - as for data in Figure $3 \mathrm{~B}$ - proved to be unsuitable to capture the geometrical regularities (i.e. both "alignment" and isometric properties) encoded by the mechanoreceptor response space (not shown). We then applied a decoding scheme based on the SRM metrics (Eqs. 5. 7), which allowed 


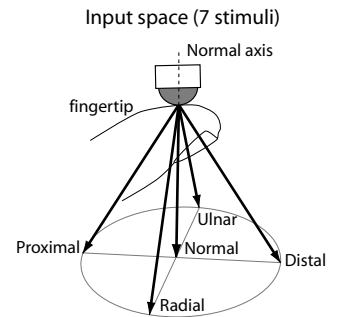

Response space

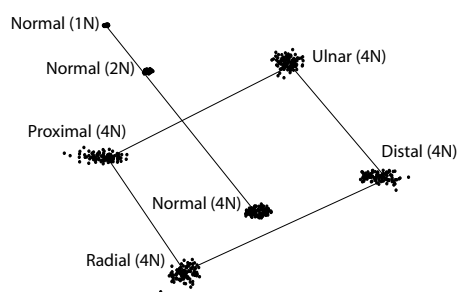

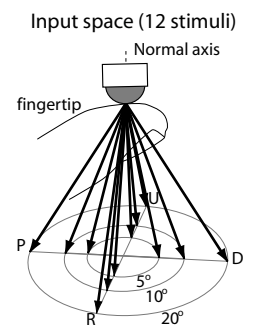

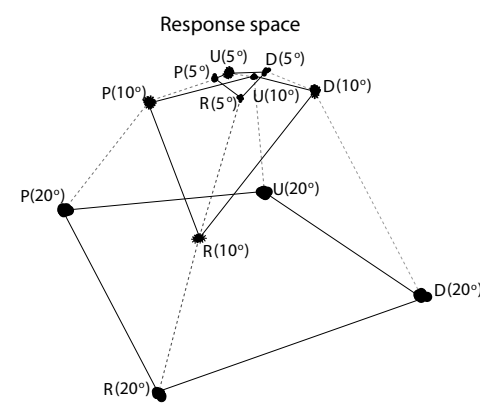

Fig. 4. Results on isometric coding. (A) Multi-dimensional scaled representation (bottom) of the metrical organisation of FA-I responses to 7 stimuli (top; i.e. a probe of fixed curvature applied along the Normal direction with force 1,2,4 N, and along the Ulnar, Radial, Distal and Proximal directions with force $4 \mathrm{~N}$ ). (B) Multi-dimensional scaled representation (bottom) of FA-I responses to 12 stimuli (top, i.e. a probe a fixed probe applied along the 4 directions Ulnar, Radial, Distal, and Proximal with different angles $5^{\circ}, 10^{\circ}, 20^{\circ}$; the force was fixed and equal to $4 \mathrm{~N}$ ).

us to mimic a population of 20 putative $2^{\text {nd }}$-order neural decoders stochastically connected to the 42 recorded mechanoreceptors. We computed the distances between responses elicited by the set of stimuli shown in Figure 44 (top) as the sum of the SRM distances for each of the 20 hypothetical $2^{\text {nd }}$-order neurons. For visualisation purposes, we applied a multi-dimensional scaling to this set of distances to determine the three dimensions that best preserved all measured distances. The projections of the multidimensional responses onto the identified 3-dimensional space are shown in Figure 4A, bottom. We obtained both isometrically-organised and "aligned" clusters in the response space. Stimulations in the Normal direction with forces 1, 2 and $4 \mathrm{~N}$ formed a line that passed orthogonally in the middle of a square formed by the responses to 4 orthogonal stimulations. Finally, Figure $4 \mathrm{~B}$ shows that isometric coding was also obtained when considering a more complex data set of 12 tactile stimuli.

\section{Discussion}

This paper highlights the importance of accounting for the metrical relations between spike trains when studying neurotransmission. We contribute to spike metrics theory by deriving a novel distance that embeds the characteristics 
(e.g. membrane time constant, threshold and slope of the non-linear firing function) of a probabilistic neural decoder. This distance helps us to determine the decoder's parameters leading to effective information transfer - in terms of both input discrimination and detection of geometrical input organisation. When decoding the activity of human FA-I mechanoreceptors we provided evidence that the temporal structure of their activity contains enough information to discriminate large number of tactile stimuli within few milliseconds and to infer geometrical relations between them. Our results suggest that first-spike latencies across multiple mechanoreceptors are relevant to rapid and non-ambiguous reconstruction of the input, whereas only by decoding entire spike train responses we can go beyond discrimination and capture higher-order properties such as isometric stimulus-response transformations. This observation may be the basis for the ability to extrapolate and/or generalise tactile perception and recognition in the presence of never experienced stimuli.

Acknowledgements. Granted by the EC project SENSOPAC (no. IST-027819IP) and by the French Medical Research Foundation (no. FDT20090916556).

\section{References}

1. Barlow, H.B.: The coding of sensory messages. In: Thorpe, W.H., Zangwill, O.L. (eds.) Current Problems in Animal Behaviour, pp. 331-360. Cambridge University Press, Cambridge (1961)

2. Brasselet, R., Johansson, R.S., Arleo, A.: Optimal context separation of spiking haptic signals by second-order somatosensory neurons. In: Bengio, Y., et al. (eds.) Adv. Neural Inf. Process Syst., vol. 22, pp. 180-188 (2009)

3. Brasselet, R., Johansson, R.S., Arleo, A.: Quantifying neurotransmission reliability through metrics based information analysis. Neural Comput. 23(4), 852-881 (2011)

4. Cover, T., Thomas, J.: Elements of information theory. Wiley, Chichester (1991)

5. Gerstner, W., Kistler, W.: Spiking Neuron Models. Cambridge Univ. Press, Cambridge (2002)

6. Johansson, R.S., Birznieks, I.: First spikes in ensembles of human tactile afferents code complex spatial fingertip events. Nat. Neurosci. 7, 170-177 (2004)

7. Johansson, R.S., Flanagan, J.R.: Coding and use of tactile signals from the fingertips in object manipulation tasks. Nat. Rev. Neurosci. 10, 345-359 (2009)

8. Kreuz, T., Haas, J.S., Morelli, A., Abarbanel, H.D.I., Politi, A.: Measuring spike train synchrony. J. Neurosci. Meth. 165, 151-161 (2007)

9. MacKay, D., McCulloch, W.: The limiting information capacity of a neuronal link. Bull. Math. Biol. 14(2), 127-135 (1952)

10. Schreiber, S., Fellous, J.M., Whitmer, S., Tiesinga, P., Sejnowski, T.: A new correlation-based measure of spike timing reliability. Neurocomput. 52-54, 925-931 (2003)

11. Shannon, C.: A mathematical theory of communication. Bell Syst. Tech. J. 27, 379-423 (1948)

12. Van Rossum, M.: A novel spike distance. Neural Comput. 13, 751-763 (2001)

13. Victor, J., Purpura, K.: Nature and precision of temporal coding in visual cortex: a metric-space analysis. J. Neurophysiol. 76, 1310-1326 (1996) 